\title{
Study of the Transition to Instability in Second- Harmonic Generation with Scale Index Method
}

\author{
Sohrab Behnia ${ }^{a^{*}}$, Javid Ziaei ${ }^{a}$, and Mehdi Khodavirdizadeh ${ }^{\text {b }}$ \\ ${ }^{\text {a}}$ Department of Physics, Urmia University of Technology, Urmia, Iran \\ ${ }^{b}$ Faculty of Electrical Engineering, Urmia University of Technology, Urmia, Iran \\ *Corresponding Author Email: s.behnia@ @ci.uut.ac.ir
}

Received: Jun. 18, 2016, Revised: Nov. 19, 2016, Accepted: Dec. 29, 2016, Available Online: Aug. 8, 2017

DOI: 10.18869 /acadpub.ijop.11.2.87

\begin{abstract}
The emergence of secondharmonic generation (SHG) is a pivotal issue to the development of nano-optical devices and interfaces. Here, we perform a classical analysis of the SHG dynamics with the aim of determining critical values of the electric field. The signals of the SHG process are nonlinear, so it seems reasonable that chaos theory can be a suitable tool to analyze their dynamics. For this purpose the bifurcation analysis and a new measure known as the scale index were employed. Obtained results show that the dynamics approaches from stable to self-pulsing and eventually to chaos through a perioddoubling route. Moreover, the scale index can detect the critical strength of the electric field, where above it the SHG dynamics is chaotic.
\end{abstract}

KEYWORDS: Bifurcation, Nonlinear optics, Second-harmonic generation, Scale index.

\section{I.INTRODUCTION}

The past decades have witnessed increasing attention on nonlinear optical phenomena [1, 2]. The first nonlinear optics experiment was performed in 1962 by Franken et al [3, 4]. Afterwards, the development of nonlinear optics has provided more robust tools on study of light matter interactions [5]. Today, the nonlinear optical phenomena are widely used for applied researches [6]. Meanwhile, frequency conversion processes play an important role in nonlinear optical phenomena such as second or higher order harmonics generations [7]. Owing to rich classical nonlinear dynamics of frequency doubling processes, the second harmonic generation
(SHG) is analyzed in this study [8]. The SHG as a frequency doubling process stands for the interaction of two photons in a nonlinear material and so the generation of one photon $[9,10]$. The main idea for applications of SHG was proposed in 1968 by Bloembergen et al [11]. Furthermore, due to being surface sensitive, SHG process provides an additional possibility to study optical properties of the nanotube surfaces [12-14].

Despite the mentioned features, having high efficiency is one of the major problems in the nonlinear optics [15-16]. We know that increasing the input field strength or the crystal length, would result in the promotion of SHG efficiency [17]. However, the stability of nonlinear optical systems is one of the most important issues in high intensity [18]. In this regard, chaos in optical systems has been reported by Savage and Walls for the first time [19-22]. Afterwards, McNeil et al. [23] have shown the self-pulsing and chaotic behavior in the SHG process inside a cavity, provided that the external electrical field to be in a sufficiently high strength. With this controversy, we study the instability of SHG process in the absence of a cavity. To this end, we apply the scale index for the first time in nonlinear optics domain. Since the chaotic behavior is a non-periodic one, for an accurate identification we need a measure to distinguish a periodic behavior from a non-periodic one. Hence, it seems that applying scale index as a non-periodic behavior identifier would help to report the transition point from stability to 
instability. To verify the results, we also plot bifurcation diagram. The rest of the manuscript was organized as follows. Section I describes the SHG dynamical model. Section II contains the obtained results, by focusing on the dynamical response of the SHG, bifurcation analysis, and the non-periodicity feature of the SHG dynamics. Finally, Section III remarks the conclusions.

\section{MODEL}

In the following we will outline the interaction in a $\chi^{(2)}$ medium. The crystal converts two "photons" of a strong pump field of frequency $\omega$ (called fundamental) into one "photon" of frequency $2 \omega$ (doubly resonant SHG). In order to achieve universal results, it is important to work with scaled parameters. Besides simplifying the numerical calculations, avoiding unnecessary errors is provided by this procedure $[8,20]$. Generally, the normalization is carried out through the loss rates of a particular cavity or the strength of the nonlinearity of the nonlinear crystal used [24]. In this regard, the normalized form of the Hamiltonian of SHG process describing coupling of electromagnetic fields at frequencies $\omega$ and $2 \omega$ in the rotating wave approximation is read as [25]:

$$
H=H_{\mathrm{int}}+H_{\text {pump }}
$$

In this approximation, rapidly oscillating terms of the Hamiltonian are neglected. The interaction Hamiltonian (in $\hbar=1$ basis) is read as:

$$
H_{\mathrm{int}}=i \frac{k}{2}\left(a^{2} b^{+}-a^{+^{2}} b\right)
$$

And the pump effect can be expressed as:

$$
H_{\text {pump }}=i\left(\varepsilon a^{+}-\varepsilon^{*} a\right)
$$

where, $k$ stands for the effective $\chi^{(2)}$ coupling strength between the two modes, with $a(b)$ being the bosonic annihilation operator for excitations at frequency $\omega(2 \omega)$, and, $\varepsilon$ is the strength of the electric field [24, 25].
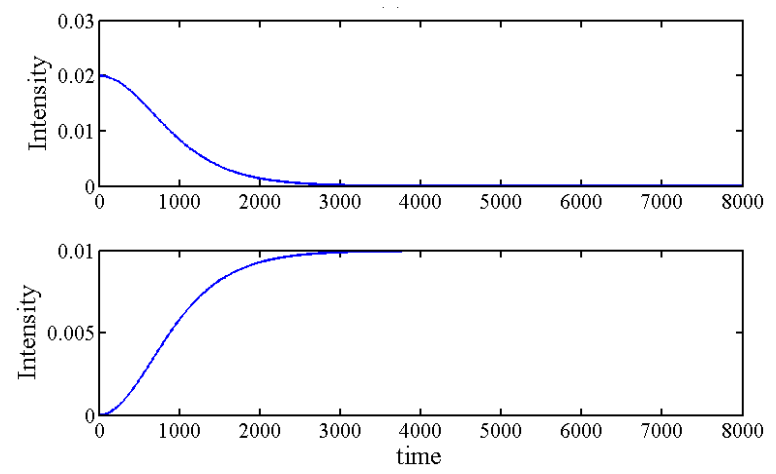

(a)
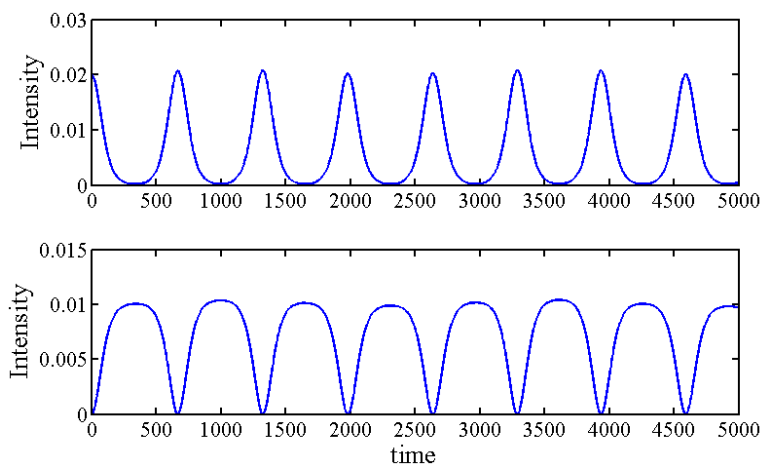

(b)

Fig. 1 Time evolution of the intensities $|a|^{2}$ (top panel) and $|b|^{2}$ (bottom panel) vs. electric field $\varepsilon$. (a) steady-state response $(\varepsilon=0)$, (b) pulsing behavior $\left(\varepsilon=10^{-6}\right)$.

Dynamical form of phase space trajectories of the Hamiltonian Eq. (3) is represented as:

$$
\left\{\begin{array}{l}
\frac{d a}{d t}=-k a^{+} b+\varepsilon \\
\frac{d b}{d t}=\frac{1}{2} k a^{2}
\end{array}\right.
$$

which is used here for classical analysis of the process.

\section{III.RESULTS AND DISCUSSION}

The electric field ${ }_{\varepsilon}$ can affect dynamics of the system. Our purpose is to find critical values which can cause the studied system to transit between stability and self-pulsing process. In this regard, we suppose the electrical field $\varepsilon$ intensity as a control parameter. 
In Fig. 1 we illustrate the time evolution of the intensities $|a|^{2}$ (top panel) and $|b|^{2}$ (bottom panel). Assuming $k=0.01 \quad$ [25] and $[0.1+i 0.1,0]$ as initial condition $[26,27]$ the classical limit Eq. (4) exhibits a period doubling transition to instability. Slowly increasing the strength of electric field reveals this function.
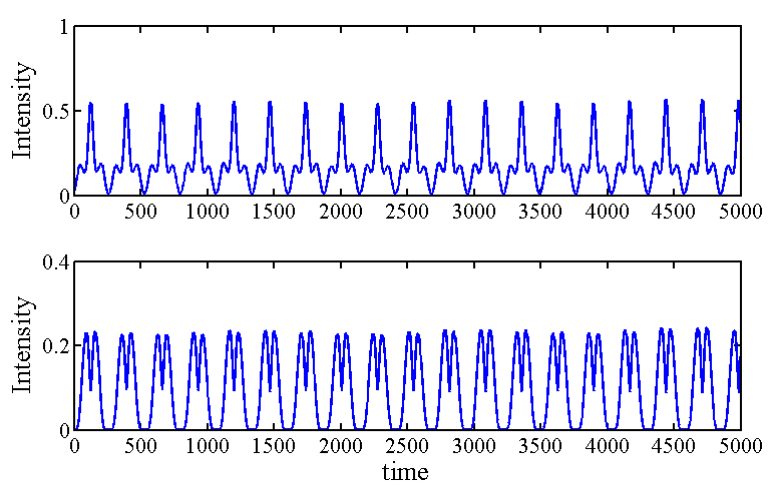

(a)
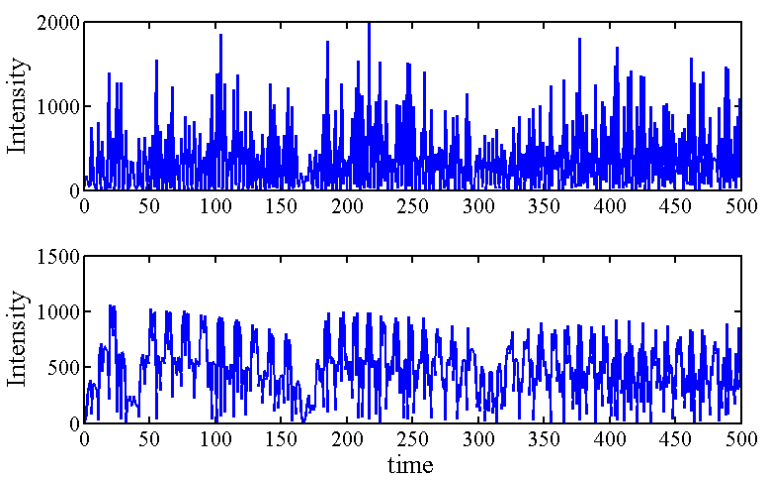

(b)

Fig. 2 Time evolution of the intensities $|a|^{2}$ (top panel) and $|b|^{2}$ (bottom panel) vs. electric field $\varepsilon$. (a) period-four cycle ( $\varepsilon=10^{-3}$ ), and (b) deep inside non-periodic regime $(\varepsilon=1)$.

Although the SHG dynamics is steady-state for $\varepsilon=0$ (Fig. 1(a)), a slight increase in the electric field will have a significant effect on the dynamics. This pattern is seen from Fig. 1(b), where the pulsing regime is dominant. Further increasing of $\varepsilon$ leads to a period doubling bifurcation and to the unstabilization of the original stable periodic cycle to a new stable period doubled cycle (Fig. 2(a)). These consecutive period doublings leads to the arising of chaos and instability, as is clear from Fig. 2(b).

The bifurcation diagrams providing a qualitative image of the system are shown in Figs. 3, where three different regions of $\varepsilon$ are presented. As is clear, small values of $\varepsilon$ does not cause a significant change in the dynamics and so small values of $\varepsilon$ corresponds with stable phases. Furthermore, increasing $\varepsilon$ leads to a period-doubling route to chaos in the classical analysis. Evidently, the system experiences successively period-doubling approach. From this view, the chaotic and unstable behaviors prevail for larger values of the $\varepsilon$ in consistent with the results of Figs. 1-2, presenting the temporal variation of intensity $|a|^{2},|b|^{2}$ for different values of the electric field. As is seen from the bifurcation diagram, the amplitude of the intensity was altered with the variation of the control parameter. This means that the parameter sensitivity of the SHG compared with the amplitude of the electric field is very high [28, 29]. This parameter sensitivity of SHG dynamics confirms previous works proving the existence of chaos in this system [20, 21, 26]. On this matter, coherently driven second-harmonic generation within a detuned Fabry-Perot cavity is predicted to exhibit self-pulsing, period doubling and optical chaos [20]. A numerical analysis of second-harmonic generation in an optical cavity pumped by an external train of pulses in the presence of damping is performed by Grygiel, et al [21]. They have reported the existence of chaos and order in the SHG dynamics by using maximal Lyapunov exponents. For the process of intracavity second-harmonic generation in the region of unstable dynamics, the presence of bifurcation and quantum delocalized states were reported by Gevorkyan [30] by studying the joint fluctuations of numbers of photons and phases of the fundamental mode and second harmonic in positive $P$ representation.

For extracting more details on the periodicity characteristics we have studied the dynamics of scale index under the variation of electric field amplitude. Non-stationary signals make 
wavelets more suitable for detecting such periodicity.

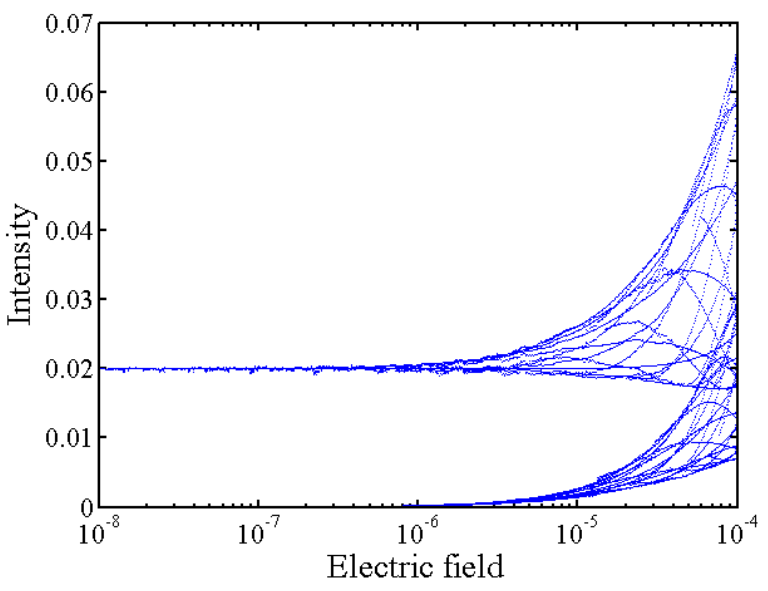

(a)

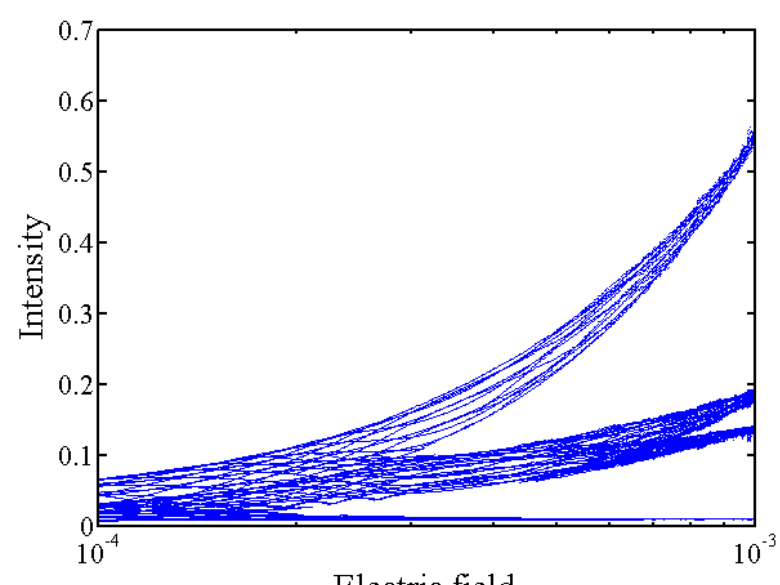

Electric field

(b)

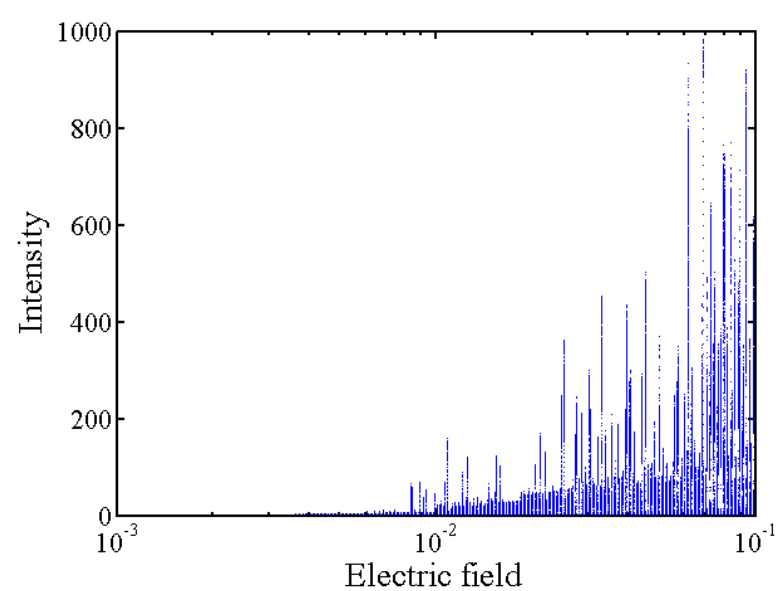

(c)

Fig. 3 Bifurcation diagram of the SHG process. (a) $\varepsilon \in\left[10^{-8}, 10^{-4}\right]$, (b) $\varepsilon \in\left[10^{-4}, 10^{-3}\right]$, (c) $\varepsilon \in\left[10^{-3}, 10^{-1}\right]$.
Moreover, wavelets have been successfully used in the analysis of many chaotic systems [31-33]. Non-periodicity as a hallmark of a chaotic behavior could be quantified through scale index analysis [33, 34]. Let the continuous wavelet transform (CWT) of a signal $f \in L^{2}$ with respect to some analyzing wavelet function $\psi$ be:

$W_{\psi} f(\tau, s)=\int_{-\infty}^{+\infty} f(t) \psi_{\tau, s}^{*}(t) d t$

where

$\psi_{\tau, s}(t)=\frac{1}{\sqrt{S}} \psi\left(\frac{t-\tau}{s}\right)$

is the wavelet basis function, $s>0$ is the scale parameter and $\tau$ is the location parameter of the wavelet. The wavelet transform is a timefrequency analysis method which differs from the more traditional short time Fourier transform by allowing arbitrarily higher localization in both time and frequency domains. The CWT does this by having a continuously varying window width. The variation of window width is controlled through what is called a scale-parameter. A large selection of localized waveforms can be employed as a transform basis rather than sinusoidal as long as they satisfy predefined mathematical criteria [35].

The periodicity analysis of a signal can be conducted through the scalogram of a function at a given scale. Then, the scalogram $S_{f}(s)$ providing the energy of signal $f$ at scale $s$, is defined as:

$S_{f}(s)=\sqrt{\int_{-\infty}^{+\infty}\left|W_{\psi} f(\tau, s)\right|^{2} d \tau}$

The scalogram is a useful tool for studying a signal, since it allows the detection of its most representative scales or frequencies. It is considered that the signal $f$ spreads over a finite time interval $I=\left[t_{i}, t_{f}\right]$. Therefore, to avoid boundary problems as a result of the overlapping of the support of $\psi_{\tau, \mathrm{s}}$ at $t=t_{i}$ or 
$t=t_{f}$, the inner scalogram of $f$ at scale $s$, $S_{f}^{\text {inner }}(s)$ comes into play:

$S_{f}^{\text {inner }}(s)=\sqrt{\int_{\tau_{i}}^{\tau_{f}}\left|W_{\psi} f(\tau, s)\right|^{2} d \tau}$

where $\left[\tau_{i}, \tau_{f}\right] \subseteq I$ is the maximal subinterval of $I$ in a way that the support of $\psi_{\tau, s}$ be included in $I$ for all $\tau \in\left[\tau_{i}, \tau_{f}\right]$.

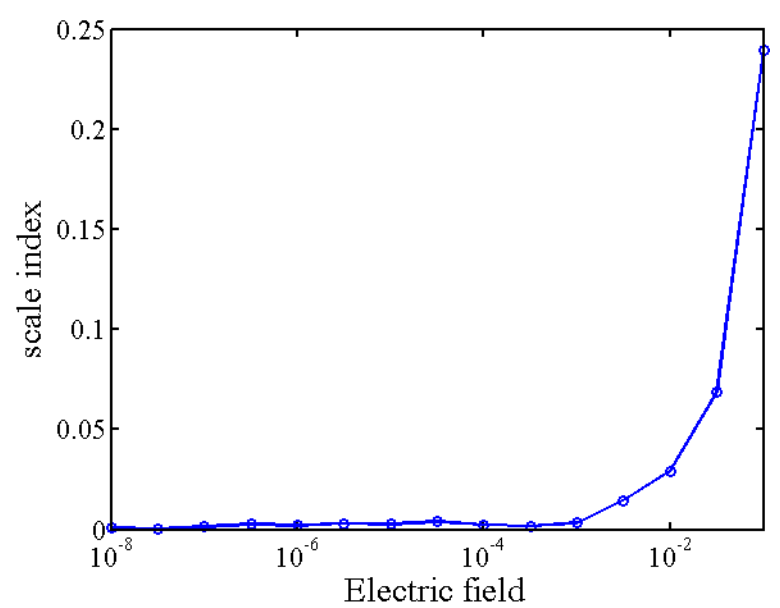

Fig. 4 The evolution of scale index under the variation of the electric field.

To compare the values of the $S_{f}^{\text {inner }}(s)$ at different scales of $s$, the inner scalogram is normalized as follows:

$\bar{S}^{\text {inner }}(s)=\frac{S_{f}^{\text {inner }}(s)}{\sqrt{\tau_{f}-\tau_{i}}}$

In practice, the computed value of inner scalogram for a finite interval $\left[s_{i}, s_{f}\right]$ comes into play. Considering $S_{\max }$ as a scale for which the inner scalogram is maximum and $S_{\min }$ as the local smallest scale, i.e., $\bar{S}^{\text {inner }}\left(s_{\min }\right) \leq \bar{S}^{\text {inner }}(s)$ for all scales $S$ included in $s_{\max } \leq s \leq s_{f}$, the scale index $i_{\text {scale }}$ of the signal $f$ is defined as:

$i_{\text {scale }}=\frac{\bar{S}^{\text {inner }}\left(s_{\text {min }}\right)}{\bar{S}^{\text {inner }}\left(s_{\max }\right)}$
An outstanding feature of scale index is its finite value, i.e., $0 \leq i_{\text {scale }} \leq 1$ : the larger the value of $i_{\text {scale }}$, the more non-periodic signal is. Regarding this bounded characteristic of scale index, it is a sensible criterion of the nonperiodicity measure. A detailed discussion about the scale index can be found in [33]. Here the selection of the scale interval $\left[s_{i}, s_{f}\right]$ is very important in the scalogram analysis. Since the non-periodic character of a signal is given by its behavior at large scales, there is no need for $s_{i}$ to be very small. In general, we can choose $s_{i}$ such that $s_{\max }=s_{i}+\varepsilon$ where $\varepsilon$ is positive and close to zero. On the other hand, $s_{f}$ should be large enough for detecting periodicities. Here, we considered the scales between $s_{i}=1$ and $s_{f}=512$.

The dynamics of scale index is shown in Fig. 4. Obviously, this analysis confirms bifurcation diagram. For small values of the electric field corresponding with stable and periodic dynamics, the scale index approaches 0 . And, larger amplitudes lead to larger values of the scale index as expected. Since temporal evolution of intensities $|a|^{2}$ and $|b|^{2}$ in weak fields are stable and show self-pulsing behavior, the high value of the scale index for high intensities indicates the disturbance of the periodicity of SHG signals. The increased value of the scale index for $\varepsilon>10^{-3}$ reflects a tendency towards more aperiodic and chaotic behavior of the SHG dynamics. This value is a critical one which separates stable and selfpulsing region from chaotic region.

\section{IV.CONCLUSION}

This work provided a computational framework for examining the chaotic effects of electric field on the SHG dynamics described mathematically by the Hamiltonian in the rotating wave approximation. Focusing mainly on the dynamical response of the SHG, the global structure of the model was studied. It was shown that the scale index is able to discern self-pulsing from chaotic regime. Moreover, the most important outcome of the present study was to show the effectiveness of 
the scale index in detecting critical value of the electric field. Furthermore, this study reveals the fact that the SHG process can be subjected to a chaotic response without any external feedback. The results of present study flashes new lights into the novel approaches that can be applied to the study of nonlinear optics. It is to be noted that the results of this study can be extended by considering the effects of the electric field in the inside of a cavity.

\section{REFERENCES}

[1] M. Nadafan, R. Malekfar, and Z. Dehghani, "Nonlinear optical properties of rigid polyurethane foam $/ \mathrm{SiO} 2$ nanocomposite," Int. J. Opt. Photon. Vol. 8, pp. 85-94, 2014.

[2] A. Capretti, E.F. Pecora, C. Forestiere, L.D. Negro, and G. Miano, "Size-dependent second-harmonic generation from gold nanoparticles," Phys. Rev. B, Vol. 89, pp. 125414 (1-5), 2014.

[3] R. Boyd, Nonlinear Optics, Academic Press, 2003.

[4] Y.D. Glinka, S. Babakiray, T.A. Johnson, M.B. Holcomb, and D. Lederman, "Resonance-type thickness dependence of optical second-harmonic generation in thin films of the topological insulator $\mathrm{Bi}_{2} \mathrm{Se}_{3}$," Phys. Rev. B, Vol. 91, pp. 195307 (1-15), 2015.

[5] A. Bonda, S. Uba, and L. Uba, "Nonlinear magnetization-induced terms in garnet film polarization in the second-harmonic generation effect: theory and experiment," Phys. Rev. B, Vol. 87, pp. 024426 (1-10), 2013.

[6] D.L. Weerawarne, X. Gao, A.L. Gaeta, and B. Shim, "Higher-order nonlinearities revisited and their effect on harmonic generation," Phys. Rev. Lett. Vol. 114, pp. 093901 (1-5), 2015.

[7] M. Lafrentz, D. Brunne, A.V. Rodina, V.V. Pavlov. R.V. Pisarev, D.R. Yakovlev, A. Bakin, and M. Bayer, "Second-harmonic generation spectroscopy of excitons in $\mathrm{ZnO}$," Phys. Rev. B, Vol. 88, pp. 235207 (1-20), 2013.

[8] S.T. Gevorkyan, G.Y. Kryuchkyan, and N.T. Muradyan, "Quantum fluctuations in unstable dissipative systems," Phys. Rev. A, Vol. 61, pp. 043805 (1-10), 2000.
[9] S. Sagadevan, "A simple theoretical approach to analyze the second harmonic generation of single crystals," Optik, Vol. 126, pp. 317-319, 2015.

[10] J. Butet, I. Russier-Antoine, C. Jonin, N. Lascoux, E. Benichou, O.J.F. Martin, and P.F. Brevet, "Universal scaling of plasmon coupling in metal nanostructures: checking the validity for higher plasmonic modes using second harmonic generation," Phys. Rev. B, Vol. 87, pp. 235437 (1-6), 2013.

[11] Y.V.G.S. Murti and C. Vijayan, Essentials of Nonlinear Optics, John Wiley, 2014.

[12] R.V. Salazar-Aparicio, R.A. V'azquez-Nava, N. Arzate, and B.S. Mendoza, "Molecular hydrogen physisorption on boron-nitride nanotubes probed by second harmonic generation," Phys. Rev. B, Vol. 90, pp. 155403 (1-5), 2014.

[13]E.A. Mamonov, I.A. Kolmychek, S. Vandendriessche, M. Hojeij, Y. Ekinci, V.K. Valev, T. Verbiest, and T.V. Murzina, "Anisotropy versus circular dichroism in second harmonic generation from fourfold symmetric arrays of G-shaped nanostructures," Phys. Rev. B, Vol. 89, pp. 121113 (1-5), 2014.

[14]M.G. Mandujano and J.A. Maytorena, "Quadrupolar second-harmonic generation by helical beams and vectorial vortices with radial or azimuthal polarization," Phys. Rev. A, Vol. 88, pp. 023811 (1-6), 2013.

[15]P. Vasudevan, S. Sankar, and S.G. Raj, "Studies on second harmonic generation efficiency of organic material 1-arginine maleate dihydrate," Optik, Vol. 124, pp. 4155-4158, 2013.

[16]C. Argyropoulos, G.D.Aguanno, and A. Al’u, "Giant second-harmonic generation efficiency and ideal phase matching with a double $\varepsilon$ near-zero cross-slit metamaterial," Phys. Rev. B, Vol. 89, pp. 235401 (1-6), 2014.

[17]H. Wang and A.M. Weiner, "Efficiency of short-pulse type-I second-harmonic generation with simultaneous spatial walk-off, temporal walk-off, and pump depletion," IEEE J. Quantum Electron., Vol. 39, pp. 937-945, 2003.

[18] Y. Silberberg and I.B. Joseph, "Instabilities, self-oscillation, anti chaos in a Simple 
nonlinear optical interaction," Phys. Rev. Lett. Vol. 48, pp. 1541-1543, 1982.

[19]J. Zhang and H. Haken, "Self-pulsing instabilities in the generation of higher harmonics," Zeitschrift für Physik B: Condensed Matter, Vol. 58, pp. 337-341, 1985.

[20]C.M. Savage and D.F. Walls, "Optical chaos in second-harmonic generation," Optica Acta, Vol. 30, pp. 557-561, 1983.

[21]K. Grygiel and P. Szlachetka, "Chaos in second-harmonic generation of light. The case of a train of pulses," Opt. Commun., Vol. 91, pp. 241-246, 1992.

[22] Y. Sen-lin, "Bifurcation and locking in an multi-quantum-well laser subjected to external injection," Opt. Commun., Vol. 282, pp. 3558-3564, 2009.

[23] K.J. Mcneil, P.D. Drummond, and D.F. Walls, "Self pulsing in second harmonic generation," Opt. Commun. Vol. 27, pp. 292-294, 1978.

[24]D.F. Walls and G.J. Milburn, Quantum Optics, Springer, 2008.

[25] M.K. Olsen, "Asymmetric Gaussian harmonic steering in second-harmonic generation," Phys. Rev. A, Vol. 88, pp. 051802 (1-4), 2013.

[26]P. Szlachetka, K. Grygiel, J. Bajer, and J. Perina, "Chaos and order in second-harmonic generation: Cumulant approach," Phys. Rev. A, Vol. 46, pp. 7311 (1-6), 1992.

[27]P.D. Drummond, K.J. McNeil, and D.F. Walls, "Non-equilibrium transitions in sub/second harmonic generation," Optica Acta, Vol. 27, pp. 321-335, 1980.

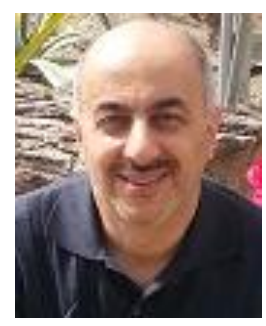

Sohrab Behnia received the M.Sc. degree in atomic and molecular physics in 1995 and the Ph.D. degree in theoretical physics, in 2000. $\mathrm{He}$ is currently the associate professor of physics in Urmia University of Technology. Dr Behnia research interests are classical and quantum chaos theories.
[28]W. Zhai, "A study of electric-field-induced second-harmonic generation in asymmetrical Gaussian potential quantum wells," Physica B, Vol. 454, pp. 50-55, 2014.

[29] J.H. Yuan, N. Chen, H. Mo, Y. Zhang, and Z.H. Zhang, "The second harmonic generation in symmetrical and asymmetrical Gaussian potential quantum wells with applied electric field," Superlattices Microstruct. Vol. 88, pp. 389-395, 2015.

[30]S.T. Gevorkyan, "Bifurcation and quantum delocalized states in second-harmonic generation," Phys. Rev. A, Vol. 62, pp. 013813 (1-7), 2000.

[31] G. Chen, S. Hsu, Y. Huang, and M.A. RoqueSol, "The spectrum of chaotic time series (II): wavelet analysis," Int. J. Bifurcation Chaos, Vol. 21, pp. 457-1467, 2011.

[32] A. Ouahabi and S. Femmam, "Wavelet-based multifractal analysis of 1-D and 2-D signals: new results," Analog Integ. Circuits Signal Process. Vol. 69, pp. 3-15, 2011.

[33]R. Benitez, V.J. Bolos, and M.E. Ramirez, "A wavelet-based tool for studying nonperiodicity," Comput. Math. Appl, Vol. 60, pp. 634-641, 2010.

[34]S. Behnia, J. Ziaei, M. Ghiassi, and A. Akhshani, "Nonlinear dynamic approach of heartbeats based on the GrudzinskiZebrowski's model," Chin. J. Phys., Vol. 53, pp. 120702 (1-17), 2015.

[35]P.S. Addison, "Wavelet transforms and the ECG: a review," Physiol. Meas. Vol. 26, pp. 155-199, 2005.

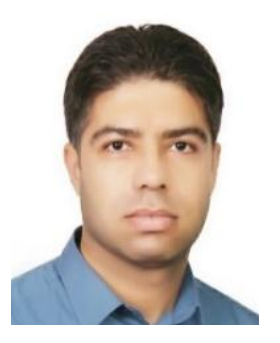

Javid Ziaei received his BSc and MSc degrees in Physics from Urmia and Tabriz Universities, Iran, in 2009 and 2011, respectively. He is currently working toward the $\mathrm{PhD}$ degree in chaos theory. His research 
interests include classic and quantum chaos theories.

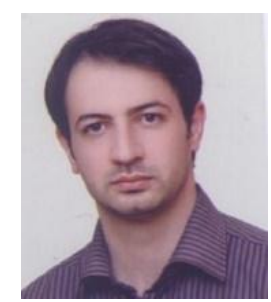

Mehdi Khodavirdizadeh was born in Urmia, in 1983. He received the M.Sc. degree in Laser Physics from Tabriz University, Iran, in 2009.
Currently he is an instructor in Electrical Engineering Faculty of Urmia University of Technology in Urmia, Iran. 